\title{
HOW SHOULD PLASTIC RECYCLATES LOOK LIKE TO BE PERCEIVED AS SUSTAINABLE: A FIRST EXPLORATION
}

\author{
Du Bois, Els (1); \\ Veelaert, Lore (1); \\ Tormans, Emiel (1); \\ Moons, Ingrid (1,2) \\ 1: University of Antwerp, faculty of Design Sciences, department of Product Development; \\ 2: University of Antwerp, faculty of Business Economics, department of Marketing
}

\begin{abstract}
Notwithstanding the positive environmental impact of recycled plastics, they are only scarcely used in new designs due to unfamiliarity and lack of material identities. This research aims to touch upon the sensorial attributes that characterise the sustainable perception of recycled plastics. Understanding this would allow to respond to the new trend of sustainable living by offering recycled materials that are successfully perceived as sustainable, and that could support the identity building of each specific recycled plastic material. Three research activities were executed to explore (i) the designerly understanding of sustainable perceived plastics; (ii) the consumer understanding of sensorial material attributes that influence the sustainable perception; (iii) the understanding of these attributes towards recycled plastic materials. Five variables were found that interfere with the perception of the participants: A weaker colour intensity, the use of colourless colours, a rougher texture, a speckled structure and the usage of a matte gloss can give a sustainable look towards a sustainable plastic material. Further research should detail these variables, its limitations and try to make defined guidelines to avoid greenwashing.
\end{abstract}

Keywords: Ecodesign, Emotional design, Sustainability, Material perception, Recycled plastics

\section{Contact:}

Du Bois, Els

University of Antwerp

Product Development

Belgium

els.dubois@uantwerpen.be 


\section{INTRODUCTION}

The market for sustainable products is growing as a reaction towards the increased consumer awareness regarding the environmental impact of plastics (Bahrudin and Aurisicchio, 2018; Bläsing and Amelung, 2017; Kanchanapibul et al., 2014; Sauerwein et al., 2017a). One of the paths to reduce the environmental impact of plastics is to recycle them and reuse the materials in new applications. Considering recycled plastics, the problem is not only to recycle it, but also to reuse recycled content (Ragaert, Delva, et al., 2017; Veelaert, Du Bois, Moons, De Pelsmacker, et al., 2020). Although recycled plastics are per definition more sustainable compared to virgin alternatives, i.e. no new resources are required and less material is discarded (Halada and Yamamoto, 2001; Vezzoli, 2014), research on recycled plastics is necessary to investigate how to enlarge its usage by increasing the knowledge of their characteristics.

Available literature covers the environmental performance of the various types of recycled plastics and their suitability as an alternative to virgin plastics (Van Kets et al., 2016; Ragaert, Hubo, et al., 2017; Vyncke et al., 2018). Despite life cycle advantages, recycled materials are not necessarily received in a positive way by either its industrial users (e.g. material engineers and designers) nor by consumers when embodied in daily products (Dehn, 2014; Karana, 2012). This is mainly as they are currently often used as "surrogate" (Rognoli, Salvia, et al., 2011) materials instead of based on their own characteristics. Similar to other emerging materials such as bioplastics, this struggle for adoption can be considered as an identity issue for recyclates (Karana, 2012; Rognoli, Salvia, et al., 2011). Even though the materials do have a sound lower environmental impact, it is down to the users' appreciation of those materials that ultimately determine their commercial success (Sauerwein et al., 2017a). This lack of identity of recycled plastics is also caused by the fact that there is not just one type of recycled plastic, but a collective for thousands of different materials (many more than the existing virgin materials due to various blends that end up in recycling processes), each having their specific technical, sensorial, emotional, economic and ecological characteristics (Veelaert, Du Bois, Moons, De Pelsmacker, et al., 2020). In order to strengthen the identity of recyclates in general, understanding and emphasizing the sustainable perception of a material would be a welcome manner of identifying the sustainable added value for the product users and would enable designers to design with it accordingly in an ethic manner. Regarding the sustainable perception of recycled plastics, it must be noticed that it is not always visible whether a material is recycled or not. Similarly, it is also not always desired by a user/consumer to know this. Nevertheless, the origin of these materials does have an impact on the perceived aesthetics and material experience, compared to their virgin (or even postindustrial) counterparts (Karana, 2012; Schifferstein and Wastiels, 2014).

\subsection{Research aim}

This article discusses an exploratory research project on the relation between consumer/user perception and different sensorial attributes of plastics. In order to make products more sustainable, it is important to understand how sustainability is perceived by consumers, and what material characteristics are driving this perception. As materials are experienced through their aesthetic/sensorial appearance, we assume that consumers' perception of sustainability is determined by specific sensorial material-related characteristics such as textures, structures, colours... In this research project, the aim is to investigate which sensorial attributes of sustainable, recycled plastics influence the consumer's perception of a sustainable product. Understanding this would allow to respond to the new trend of sustainable living by offering recycled materials that are successfully perceived as sustainable, and that could support the identity building of each specific recycled plastic material.

In practice, the sensorial attributes are investigated with the aim to define the characteristics of a recycled plastic material that is perceived as sustainable. The novelty of this research is that we do not want to identify the characteristics of specific materials, but aim to understand how a theoretically sustainable perceived (recycled) plastic material is characterised. This understanding would be of interest to further extend and extrapolate to other materials and their specific production techniques and surface treatments. To structure the research, the sensorial attributes on a material level of Karana (Karana, 2009) are used. Consequently, in order to be able to define the ideal sustainable perceived material, the general research question is 'How do sensorial attributes of sustainable recycled plastics influence its sustainable perception by consumers?' 


\subsection{Problem of sustainable perception versus real sustainability}

Many actions are taken to achieve a sustainable product perception (Pallavi and Banerjee, 2017): logos, branding, labels, colours, ... but also the look of the material and the use of recycled plastic materials in sustainable products. Within this research, we will not investigate the needed logos, brandings or labelling, but we are only interested in understanding the sustainable perception of materials, especially plastics, and the necessary look of the material to be perceived as sustainable. Existing research learned that many sustainably branded products nowadays use natural materials like wood and natural fibres (Karana and Nijkamp, 2014; Osburg et al., 2016). Consequently, a very common trend in sustainable products is the use of composite materials (Rognoli, Karana, et al., 2011) in which a mixture of plastics with natural compounds is made. By using the natural materials, the product is still perceived as ecological and sustainable, but the technical aspects will be better as the plastic enables higher flexibility in shaping, production... . However, in contrast to this natural, sustainable perception, composite products are hard to recycle because the natural compound links with the plastic and it is difficult to separate them (Carus et al., 2008). Moreover, they are often made from thermosets, which cannot be mechanically recycled. This results in a low actual sustainability, which is in high contrast with the perception. The question is whether this executional greenwashing is done by purpose or due to lack of sufficient insights (Delmas et al., 2010).

In contrast with sustainably perceived products, the opposite is also existing. These products are from a sustainability or circular economy perspective actually very interesting, but they are not perceived as sustainable by consumers, nor are the used materials. A typical example of this type of products is a beer crate. Made out of PE, these beer crates rotate in an efficient collection system in which they are washed and reused continuously. Moreover, if they get damaged, the existing collection system and the monomaterial of the crate ensures gathering of pure materials and efficient recycling into new crates or other products). However, the often colourful and shiny plastic material of the crates does not increase the sustainable perception of its users regarding to the product. In Figure 1, this duality between perceived and actual sustainability is shown to clarify the aim of the research.

\subsection{Sensorial attributes to characterize materials}

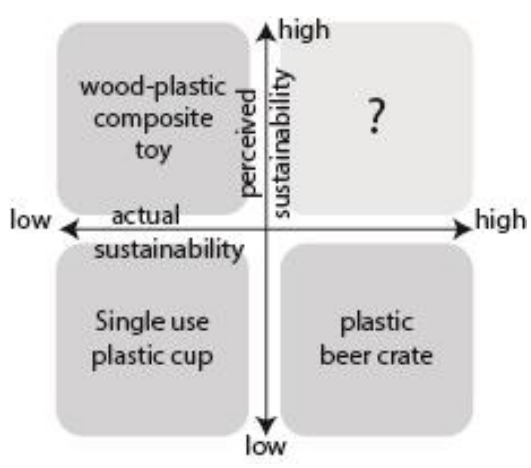

Figure 1. relation between actual and perceived perception of products/materials and some specific examples (question mark refers to products/materials of interest)

As sustainable perception of materials is mostly determined by the experience(s) the material evokes with people, we need to approach recycled plastics from a user-centred perspective(Heidbreder et al., 2019). Insights are needed to understand which intangible or sensorial aspects of materials (Karana, 2009; Zhou et al., 2008) could explain the sustainable perception of materials. It is known that sensorial attributes such as texture and colour influence the consumer in his/her perception of a material (Karana et al., 2014). While the environmental performance and technical functionalities have been examined to great extent by industry and academia (Ashby and Johnson, 2013; Pallavi and Banerjee, 2017), in contrast, only recently research is done to start investigating the user-centred or experiential perspective of materials. Still very few experiential insights and descriptive data on materials is available, whereas this knowledge would facilitate designers to construct effective strategies to manipulate meaning-creation which is needed to increase valorisation of recycled plastics in the design of new products (Sauerwein et al., 2017b). However, a solid understanding of how usercentred material characteristics should be measured is only getting formed (Veelaert, Du Bois, Moons and Karana, 2020) and can be used in this research. We will limit this research towards the understanding of only the sensorial user-centred attributes amongst all others.

\section{RESEARCH STRUCTURE}

In order to explore the general research question of "what sensorial attributes determine the sustainable perception of recycled plastics for consumers?", three studies were executed to identify these attributes and further test their optimal appearance. In Figure 2, an overview is shown of the different research actions, their aim and their reasoning towards the general research question. In each of the three smaller 
studies, different methods are used and different sub-questions are answered. Each study builds upon the results of the previous study, in order to deepen the understanding.

The first study focusses on the perception of the designer to understand the sustainable perception of daily-life products in general, without any limitation to materials. Designers were selected as specific target participants in this study since they are considered to be experts in thinking, reasoning and selecting materials, and we aim to receive better professional insights and argued answers. We expect designers to come up with more ideas about sensorial attributes of a material as they are assumed to understand material qualities and the different factors that influence material selection in the design process (Karana, 2009). The second study is done with end-consumers and examines which sensorial attributes affect the consumer's choice in sustainable products. Here, we aim to relate the perception of the consumer to the perception of the designer from the first study, with a specific focus to materialrelated sustainability perception. In the third study, the translation is done towards recycled plastic materials. The focus is put on sensorial and experiential attributes by touching and seeing specific newly designed materials.

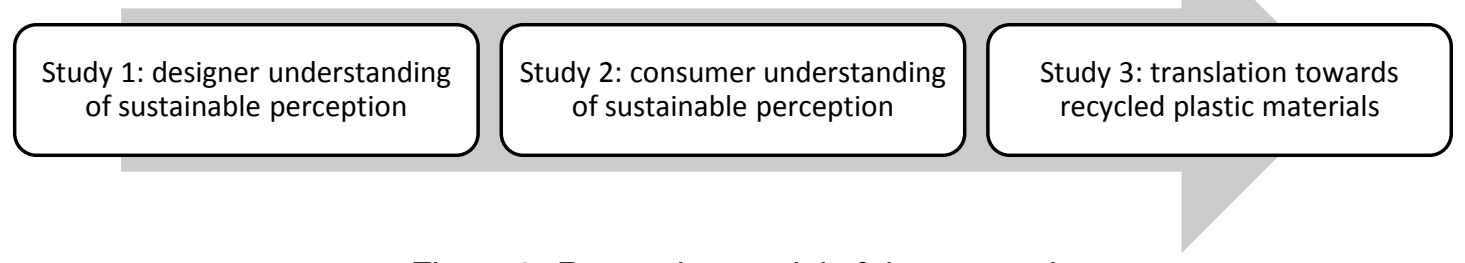

Figure 2. Reasoning model of the research

\section{STUDY 1: DESIGNER UNDERSTANDING OF SUSTAINABLE PERCEPTION}

\subsection{Aim, material and methods}

The aim of study 1 was to identify the different variables for sustainable perception in a qualitative manner. This first exploratory study was done by understanding the sustainable perception of daily-life products. All participants were designers, design students, or had a clear connection with the design world. The study was carried out by the use of Instagram, a popular photo and video sharing social networking service. This novel approach of data collection gives the opportunity to receive different answers quickly using visual information (Lee et al., 2015).

During a preliminary investigation, multiple plastic products were selected. All chosen products were branded as sustainable (by their producers) (using sustainable branding and labels, the look of the material, and/or the use of recycled plastic materials), whereas the actual sustainability of the material could be sometimes questioned. The 10 selected products are (1) a reusable bottle (Dopper, n.d.), (2) the Unusual chair van Planq design studio(PLANQ., n.d.), (3) Seepje washing product (Seepje, n.d.), (4) cup from bioplastics (Dynamec, n.d.), (5) Elho watering can made from recycled PP (Elho, n.d.), (6) Dove Original Liquid Soap Refill (Dove, n.d.), (7) reusable coffee cup made from bamboo composite material (BALEV BIO, n.d.), (8) Reusable Silicone Food Storage Bags (Stasher, n.d.), (9) reusable plastic straws (Etsy, n.d.), (10) Method soap dispenser (Method, n.d.). More information on the products is shown in Table 1. The actual sustainability of the material is reasoned by the researchers based on the origin of the material (bio or not),material lifetime, recyclability, recycled material, non-composite redetermined by 3 or more positive qualitative evaluations. It should be noted that no sustainable behaviour nor product sustainability is included in this evaluation. This evaluation can be discussed but is only indicative in the study

Respondents were asked to follow a specific Instagram profile. Using the poll-function, a picture of one of the selected products was shown with the question if the product was sustainable or not. After filling in the poll, a next board with the same picture is given with an open why-question. In a period of 17 days, 10 pictures of products were posted. Table 1 gives a representation of the different stories and different numbers of respondents for each question. This data was semantically organized into different sustainable variables. The responses were processed by means of frequency tables and content analysis. The results of the first question were counted (number of yes and no answers), whereas the second 'open-ended why' question was processed by means of a content analysis and organisation into semantic categories. 
Table 1. overview of the selected products and the responses of the study per product

\begin{tabular}{|c|c|c|c|c|c|c|c|c|c|c|}
\hline \multirow{2}{*}{$\frac{\text { ID }}{\text { Product photo }}$} & 1 & 2 & 3 & 4 & 5 & 6 & 7 & 8 & 9 & 10 \\
\hline & s. & & Tin & & & $=$ & Fु, & & & \\
\hline Material origine (bio?) & - & - & - & + & - & - & +- & - & + & - \\
\hline Material lifetime & + & + & - & - & + & - & + & + & +- & - \\
\hline Recycled material & - & + & + & - & + & - & - & - & - & + \\
\hline Recyclable & + & - & + & - & + & - & - & - & + & + \\
\hline Non-composite material & + & - & + & + & + & + & - & + & + & + \\
\hline Reasoned material sustainability & + & - & + & - & + & - & - & - & + & + \\
\hline Valid answers poll & 40 & 69 & 49 & 48 & 41 & 32 & 37 & 38 & 29 & 30 \\
\hline Valid answers open question & 18 & 48 & 34 & 30 & 23 & 25 & 20 & 29 & 32 & 20 \\
\hline
\end{tabular}

\subsection{Results of study 1}

The results that were found in this study could be split into two different outcomes. First of all, the first poll-question gives more information about the perceived sustainable character of the different products. Figure 3 gives an impression of the relation between the actual sustainability of the product (based on the outcome of Table 1) and the perceived sustainability, based on the first question asked in the Instagram study. Doing so, it was possible to have an overview and creating some new insights in the perception of a designer towards the product and the material that is being used.

Based on the second question, the following different semantic categories of variables were identified that clarify the sustainable perception (of whom the bold are material-related):

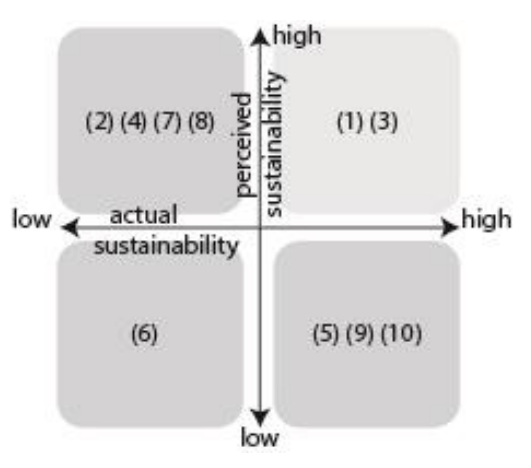

Figure 3. Results of the first question of Study 1

- Reusability and product lifetime (e.g. "The product can be reused"); total occurrence: 116

- Use of recycled materials (e.g. "The material looked as a recycled one."); total: 92

- Reference to natural materials (e.g. "It looks like a material that is natural based."); total: 49

- Colour, ethos (e.g. "The colour of the product is sober, in that way it is more sustainable."); total: 42

- $\quad$ Functional features (e.g. "I think the material is not strong enough for a long time."); total: 38

- Creation of waste (e.g. "I cannot throw it away in a recycle bag, because it contains materials that cannot be recycled.); total: 55

- Textual communication (e.g. "The claim on the product refers to the sustainability of the product."); total: 32

- Use of glue/resin (e.g. "The product contains multiple glues to connect al parts."); total: 22

- Structure/Texture (e.g. "The speckles in the material refer to a sustainable material."); total: 20

- Number of parts (e.g. "The number of parts with different materials is too large."); total: 5

The positive or negative influence of the variable was not taken into account, as for the moment, we are only interested to get an overview of all variables that influence the perceived sustainable perception. This study only gave a first insight in which variables could have an influence on the perception of designers, the study was not large enough to be representative and can consequently not be generalized. 


\section{STUDY 2: CONSUMER UNDERSTANDING OF SUSTAINABLE PERCEIVED MATERIAL ATTRIBUTES}

\subsection{Material and methods}

Study 2 focusses on the consumers' perception of sustainable materials and builds upon the attributes identified in study 1 . To explore the consumers perception, an online survey was done to question both the sustainable perception of a product and its material. For this study, a group of people between 16 and 25 years old were targeted that did not had any link with the broad design field. The study was distributed using snowball sampling. In total, 172 respondents completed the survey. The aim of the study is to identify the set of sustainable material attributes that influences the sustainable perception of a material.

An online survey existed of two parts: (i) as a warming up exercise, the respondents were asked to categorize 15 products and place them in three different frames: sustainable, no opinion and nonsustainable. Some of the products were also used in the first study, the other products were selected by the same criteria in study 1 . (ii) Next, the respondents were asked to select how each (materialrelated) attributes influences their choice of sustainability of a product, using a five points scale.

To start, the list of sensorial scales of (Karana et al., 2009) was taken as basis. A first, evaluation and shortening was done of this list, since the format of an online-survey does not allow to evaluate all 17 attributes, e.g. the acoustics of the material could not be defined in an online survey. In a preliminary discussion with design experts $(n=11)$, eight attributes were selected that could be used in the online study: (i) colour intensity (saturation), (ii) colourfulness (amount of colours), (iii) texture (material surface appearance), (iv) structure (speckles in the material), (v) transparency (see-trough), (vi) scratchability (visually scratchable), (vii) ductility (plastic deformation), and (viii) glossiness (shininess). Structure is an attribute that was originally not in the original scale, but it was added, based on the results from Study 1. This list of eight sensorial attributes was used in the form of a fivepoint bipolar semantic scale.

\subsection{Results}

Respondents were asked to select the attributes that mostly relate to the sustainability perception according to their perspective, and to identify for each attribute what the ideal sustainability level is. In Figure 4, the different answers and the means per attribute are shown.

Regarding the transparency, no probable relation with sustainable perception could be found, nor can we conclude anything regarding scratchability and ductility as their mean is between 2,50 and 3,50. On the other hand, colour intensity, colourfulness, glossiness, texture and structure do indicate that they are specific for sustainable perceived materials. From this survey, we can conclude from a theoretical perspective that the respondents consider a sustainable material to have a weaker colour intensity, rather colourless, matte surface, rough texture and many speckles.
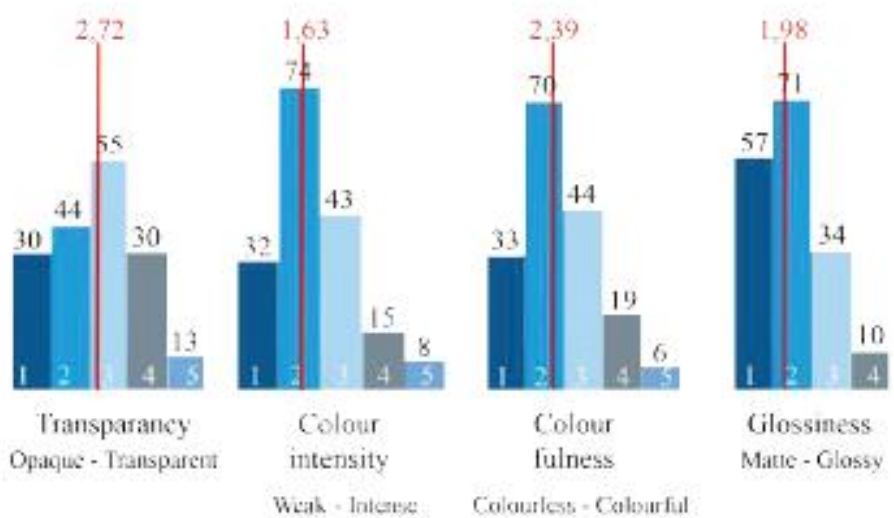

lulness

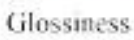

Matte - Glossy

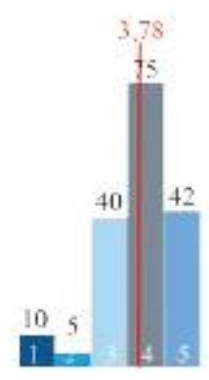

Texture

Smooth-Rough

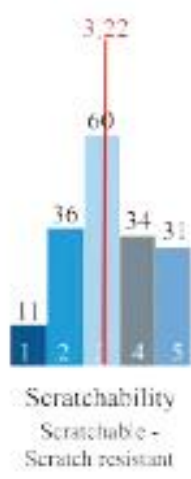

Colourless - Calsurful
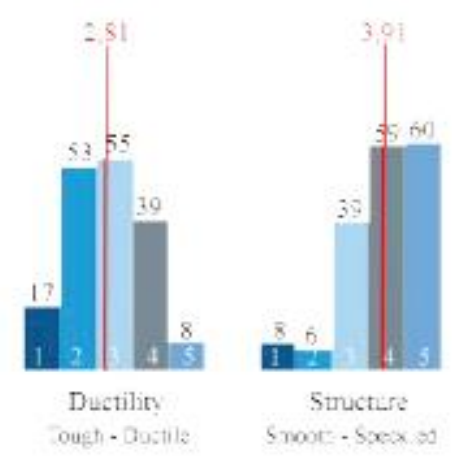

Figure 4. Results of the different sensorial material attributes that define sustainability perception (mean is added with a red line) 


\section{STUDY 3: TRANSLATION TOWARDS SUSTAINABLE RECYCLED PLASTICS}

\subsection{Materials and methods}

In the third study, the results of the first and second study are translated into practical material samples of recycled plastics in order to eliminate the product context and answer the research sub question: 'Which material attributes influence the consumers' perception of sustainability during physical interaction with new (recycled) materials?'. For example, based on study 2, we learned that the glossiness on the surface of a material has an influence on the sustainable perception of a product. Here, the ideal degree of glossiness is questioned to achieve highest possible sustainable perception. The technique of projective mapping (Varela and Ares, 2016) was considered to be useful for sensory analysis with real-life samples.

Study 3 also includes haptic sensorial experience to influence the sustainable perception of a consumer. Therefore, physical material samples were made of recycled polypropylene and were made specifically to examine one variable (shown in Figure 5-6). Polypropylene was chosen as the researchers could have access to a wide variety of these recycled pellets and they could be relatively easy handled without any risk. However, the type of plastic material is not in our interest in this study. The samples were made in house, without any specific production facility, so just by selecting and melting a specific mixture of pellets. In that way, it was possible to create samples that vary on one attribute only at the time. For each attribute, a set of six or seven new samples were used. These sets were given to the participants to explore the ideal sustainable perception level of all five attributes (Colour intensity, colourfulness, texture, structure and glossiness) and reason upon the sensorial attributes of an ideal sustainable perceived recycled plastic. Each attribute was highlighted in different grades and the participants were asked to map each set on two different scales. The x-axis holds the sensorial material attribute. The y-scale was fixed and included the factor non-sustainable and sustainable.

This study contains 25 respondents, again all being incorporated in the target audience from 18 to 26 years of age and non-designers. Photographs were taken, and the coordinates of each sample were registered in an excel spreadsheet. Afterwards, a multiple factor analysis was done for each variable with SPSS, according to the projective mapping technique. A scatterplot was made to explore the relation between the different samples. The mean was calculated for each attribute and so it was possible to understood which sample is more sustainable than another for each variable. In the last part of the study, the participant had to answer three other open-ended why-questions to figure out by which attribute he/she was influenced most to select something sustainable. And also, which attribute has no influence on the sustainability of the samples.

\subsection{Results}

Texture - (Figure 5) a slight increase in the line points that might indicate that a rougher texture is perceived more sustainable. However, there is no clear indication that a smoother or rougher material influences the sustainable perception. Similarly, during the why questions, from the 25 respondents, 16 respond that the attribute texture is considered to have the least influence on sustainable perception.

Colour intensity - ( Figure 6a) shows a clear correlation with sustainable perception, having a weaker colour is perceived as more sustainable. Products that were more intense in colour were pointed as less sustainable. In the why- questions, 2 (out of 25) respondents consider the colour intensity as the most determining attribute for sustainability perception. Consequently, we can conclude that colour intensity needs to be considered for sustainable perception.

Colourfulness - (Figure 6b) there is a soft relation between colourfulness and sustainable perception. Colourless materials appeared to be perceived as slightly

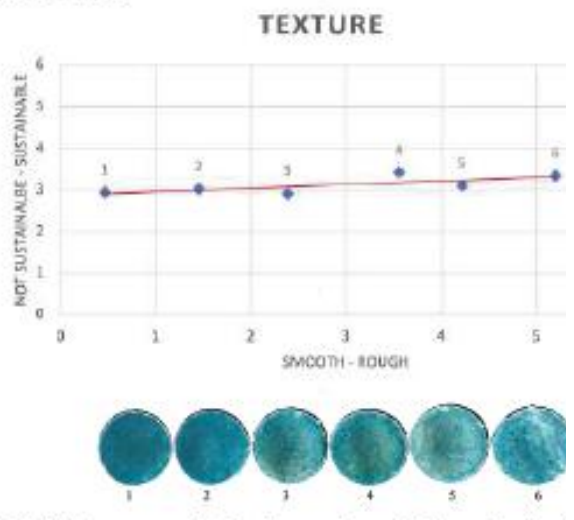

Figure 5. the attribute texture with used samples. 
more sustainable compared to heavy coloured variants. However, based upon the why questions, 7 respond out of 25 argued that the variable colourfulness has least influence on the sustainability perception.

Glossiness - (Figure 6c), is perceived more sustainable when the material is more matte. Also in the additional why-questions, out of 25 respondents, 5 respond responded that the variable glossiness has the largest influence on their perception of sustainability. Consequently, glossiness needs to be considered for sustainability perception. This glossiness might not only be material related but can also be influenced by the production technique and surface finishing, and can consequently be easily modified accordingly.

Structure - (Figure 6d), at first sight, for Structure there seemed to be no relation with the sustainable perception. However, looking at the data in more detail, we see that respondents did respond in two opposite ways to this. By calculating the mean, these extremes are not visible anymore. Also in the additional why-questions, out of 25 respondents, 18 respond argues that the structure mostly determines the sustainable perception. Some participants are more likely to see the most speckled materials as the most sustainable. Another group of participants argued that the smoothest, as a more sustainable material, because they mentioned that products which are too speckled, contain more different kinds of materials in one material, so the material is not sustainable. Consequently, we can conclude that more research is needed to understand this polarity in the correlation of structure and sustainable perception.

\section{DISCUSSION AND CONCLUSION}

This research is a step forward towards understanding sustainable perception of materials and identify the influential material variables. The presented research tried to examine different material attributes that have an influence on the sustainable perception of plastic products. Evaluating sustainable perception was found to be not evident. The separation of the research in three parts helps to identify variables that can be related to material characteristics, such as sensorial attributes. Five variables were found that interfere with the sustainable perception of the participants: A weaker colour intensity, use of colourless colours, a rougher texture, a speckled structure and the usage of a matte gloss can give a sustainable look towards a sustainable plastic material. Further research should detail these variables and try to make defined guidelines to bring these variables in a sustainable selection tool for plastic materials. If these guidelines are defined in the design process to market sustainable materials and products, the greenwashing practices could be reduced.

The research was limited by specific aspects. However, by executing the research some learnings popped up that are useful for further investigation of the domain.
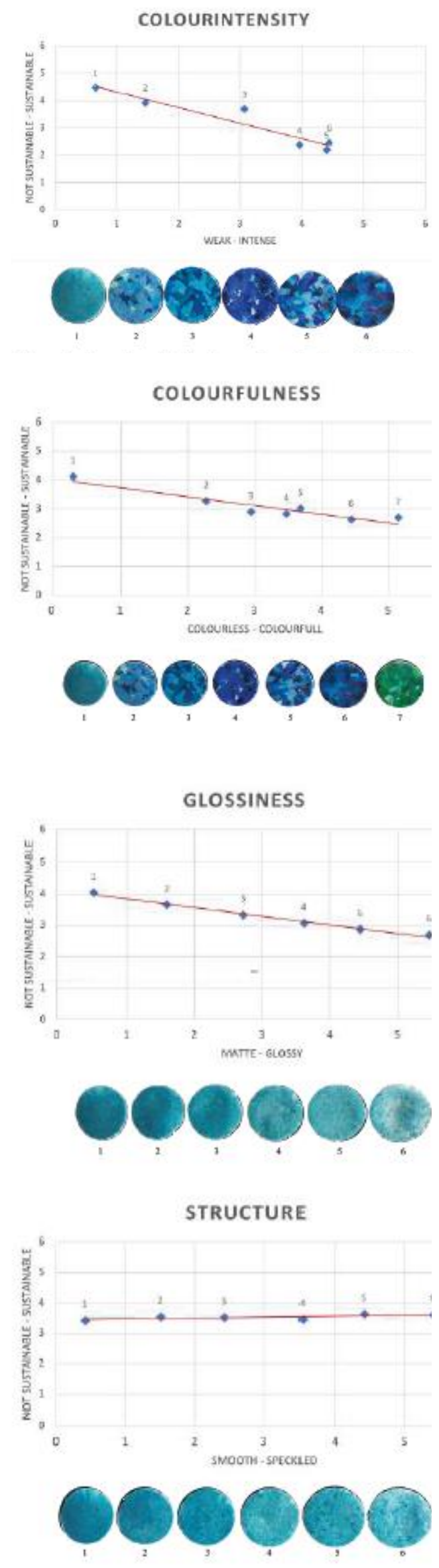

Figure 6. the attributes with used samples. (a) colour intensity, (b) colour fulness, (c) glossiness, (d) structure 
- Importance of excluding the context of the material usage. Material perception does not stand as such but can be an enabler to strengthen the perception of the user regarding sustainability perception. Also the opposite was found as it was mentioned in Study 1, the product's lifespan and reusability also have an influence on its sustainable perception. Although, this variable cannot be used on material level, it does affect the perception of the material. Consequently, in further research it would be advised to use product and context independent samples.

- The use of Instagram as a research medium, proved to be a useful tool to capture quick responses, to create an interaction with the respondents: it increased the intuitiveness and straightforwardness of the respondent. On the other hand, the use of Instagram has a few disadvantages such as the 24 hours a story was showed and then disappeared, the manual work to describe all the answers and collect them in a spreadsheet. In addition, it should also be noted that online testing reduces the ability to touch and feel the material, which is considered to be an important sensorial variable.

- Importance of danger of misusing it to create greenwashing. We must be cautious that such variables for sustainable perceived materials can also be used to greenwash other materials that are not sustainable and create the wrong impression towards consumers. Further research should try to protect these variables against greenwashing practices. In contradiction, these variables could be used to clarify the sustainability of sustainable materials in a more specific manner.

This research was only a starting point to touch upon possible variables that unconsciously effect the sustainable perception of materials. Further research is essential to implement this in sustainable material selection-tools.

\section{ACKNOWLEDGMENTS}

We are grateful to the support offered by Peter Segers from SUEZ Belgium and QCP.

\section{REFERENCES}

Ashby, M. and Johnson, K. (2013), Materials and Design: The Art and Science of Material Selection in Product Design.

Bahrudin, F.I. and Aurisicchio, M. (2018), “'Is this wallet made of real leaves?': A Study of Sustainable Materials’ Emotional Experiences”, DS 91: Proceedings of NordDesign 2018, Linköping, Sweden.

BALEV BIO. (n.d.). "Bamboo Cups and Bamboo Kitchenware”, available at: https://bamboo-cup.eu/en/ (accessed 11 December 2020).

Bläsing, M. and Amelung, W. (2017), "Plastics in soil: Analytical methods and possible sources", Science of the Total Environment, Vol. 612, pp. 422-435.

Carus, M., Gahle, C. and Korte, H. (2008), "Market and future trends for wood-polymer composites in Europe: The example of Germany", Wood-Polymer Composites, Woodhead Publishing, pp. 300-330.

Dehn, J. (2014), "Conception and realization of a sustainable materials library", in Karana, E., Pedgley, O. and Rognoli, V. (Eds.), Materials Experience: Fundamentals of Materials and Design, Butterworth-Heinemann, Oxford, UK, pp. 155-168.

Delmas, M.A., Burbano, V.C., Furlow, N.E., Guide, P., Marketing, G.G., Maier, C.D., Matthes, S., et al. (2010), "From Greenwash to Great .", The International Communication Gazette, Vol. 34 No. 12, pp. 223-249.

Dopper. (n.d.). "Waterfles met een Missie Dopper", available at: https://dopper.com/nl (accessed 11 Dec 2020)

Dove. (n.d.). "Dove caring hand wash original liquid soap refill", available at: https://www.parfumdeo.nl/ 4000388179004-dove-caring-hand-wash-original-handzeep-navulling-flacon-500-ml.html (accessed 11 December 2020).

Dynamec. (n.d.). "PLA Compostable Cold Cups, Green Tree", available at: http://dynamec.com.cy/store/placompostable-cold-cups-green-tree (accessed 11 December 2020).

Elho. (n.d.). "green basics gieter 10ltr", available at: https://www.elho.com/be/collectie/product/8711904302764/ gb-watering-can-101-lime-green/?s=500 (accessed 11 December 2020).

Etsy. (n.d.). "Herbruikbare rietjes BPA vrije Eco vriendelijke gestreept", available at: https://www.etsy.com/nl/listing/153128410/herbruikbare-rietjes-bpa-vrije-eco (accessed 11 Dec 2020).

Halada, K. and Yamamoto, R. (2001), "The current status of research and ecomaterials", MRS Bulletin, Vol. 11, pp. 871-879.

Heidbreder, L.M., Bablok, I., Drews, S. and Menzel, C. (2019), “Tackling the plastic problem: A review on perceptions, behaviors, and interventions", Science of The Total Environment, Elsevier, Vol. 668, pp. 1077-1093.

Kanchanapibul, M., Lacka, E., Wang, X. and Chan, H.K. (2014), “An empirical investigation of green purchase behaviour among the young generation", Journal of Cleaner Production, Vol. 66, pp. 528-536.

Karana, E. (2009), Meaning of Materials, Delft University of Technology, Delft, the Netherlands. 
Karana, E. (2012), “Characterization of 'natural' and 'high-quality' materials to improve perception of bioplastics", Journal of Cleaner Production, Vol. 37, pp. 316-325.

Karana, E., Hekkert, P. and Kandachar, P. (2009), "Meanings of materials through sensorial properties and manufacturing processes", Materials \& Design, Elsevier, Vol. 30 No. 7, pp. 2778-2784.

Karana, E. and Nijkamp, N. (2014), "Fiberness, reflectiveness and roughness in the characterization of natural and high quality materials", Journal of Cleaner Production, Elsevier Ltd, Vol. 68, pp. 252-260.

Karana, E., Pedgley, O. and Rognoli, V. (2014), Materials Experience: Fundamentals of Materials and Design, Butterworth-Heinemann, Oxford, UK.

Van Kets, K., Van Damme, N., Delva, L. and Ragaert, K. (2016), "The effect of the compatibilizer SEBS-gGMA on the blend PP-PET: virgin and recycled materials", PPS 32.

Lee, E., Lee, J.-A., Ho Moon, J. and Sung, Y. (2015), "Pictures Speak Louder than Words: Motivations for Using Instagram", available at:https://doi.org/10.1089/cyber.2015.0157.

Method. (n.d.). "hand soap dispenser", available at: https://methodhome.com/product-category/hand/ (accessed 11 December 2020).

Osburg, V.-S., Strack, M. and Toporowski, W. (2016), "Consumer acceptance of Wood-Polymer Composites: a conjoint analytical approach with a focus on innovative and environmentally concerned consumers", Journal of Cleaner Production, Vol. 110, pp. 180-190.

Pallavi, Y. and Banerjee, S. (2017), "Exploring material selection using”, No. January.

PLANQ. (n.d.). "Unusual Chair”, available at: https://www.planqproducts.com/unusualchair (accessed 11 December 2020).

Ragaert, K., Delva, L. and Van Geem, K. (2017), "Mechanical and chemical recycling of solid plastic waste", Waste Management, Elsevier, Vol. 69, pp. 24-58.

Ragaert, K., Hubo, S., Delva, L., Veelaert, L. and Du Bois, E. (2017), “Upcycling of contaminated postindustrial polypropylene waste: A design from recycling case study", Polymer Engineering \& Science.

Rognoli, V., Karana, E. and Pedgley, O. (2011), "Natural fibre composites in product design: An investigation into material perception and acceptance”, DPPI'11 - Designing Pleasurable Products and Interfaces, Proceedings, ACM Press, Milan, Italy, 22-25th June, p. 1.

Rognoli, V., Salvia, G. and Levi, M. (2011), “The aesthetic of interaction with materials for design”, Proceedings of the 2011 Conference on Designing Pleasurable Products and Interfaces - DPPI '11, ACM Press, New York, New York, USA, p. 1.

Sauerwein, M., Karana, E. and Rognoli, V. (2017a), "Revived Beauty : Research into Aesthetic Appreciation of Materials to Valorise sustainability Revived Beauty : Research into Aesthetic Appreciation of Materials to Valorise Materials from Waste", No. April, available at:https://doi.org/10.3390/su9040529.

Sauerwein, M., Karana, E. and Rognoli, V. (2017b), "Revived Beauty: Research into Aesthetic Appreciation of Materials to Valorise Materials from Waste", Sustainability, Multidisciplinary Digital Publishing Institute, Vol. 9 No. 4, p. 529.

Schifferstein, H. and Wastiels, L. (2014), "Sensing materials: Exploring the building blocks for experiential design”, in Karana, E., Pedgley, O., Rognoli, V. (Ed.), Materials Experience: Fundamentals of Materials and Design, 1st ed., Butterworth Heinemann, Oxford, pp. 15-26.

Seepje. (n.d.). "Natuurlijk wasmiddel, poetsmiddel en handzeep - Seepje”, available at: https://www.seepje.nl/ (accessed 11 December 2020).

Stasher. (n.d.). "Reusable Silicone Bags | Reusable Snack, Sandwich \& Storage Bags - Stasher", available at: https://www.stasherbag.com/ (accessed 11 December 2020).

Varela, P. and Ares, G. (2016), Novel Techniques in Sensory Characterization and Consumer Profiling, Journal of Neurochemistry, Vol. 88, CRC press, available at:https://doi.org/10.1046/j.1474-1644.2003.2314p28_01.x.

Veelaert, L., Du Bois, E., Moons, I. and Karana, E. (2020), "Experiential characterization of materials in product design: A literature review”, Materials \& Design, Elsevier, Vol. 190, p. 108543.

Veelaert, L., Du Bois, E., Moons, I., De Pelsmacker, P., Hubo, S. and Ragaert, K. (2020), “The Identity of Recycled Plastics: A Vocabulary of Perception”, Sustainability, Multidisciplinary Digital Publishing Institute, Vol. 12 No. 5, p. 1953.

Vezzoli, C. (2014), “The 'Material' Side of Design for Sustainability”, in Karana, E., Pedgley, O. and Rognoli, V. (Eds.), Materials Experience: Fundamentals of Materials and Design, Butterworth-Heinemann, Oxford, UK, pp. 105-121.

Vyncke, G., Onnekink, J., Feenstra, T. and Ragaert, K. (2018), "Design from Recycling for post-consumer WEEE plastics", International Conference on Polymers and Moulds Innovations (PMI2018), Ghent, Belgium, p. 6.

Zhou, C.-C., Yin, G.-F. and Hu, X.-B. (2008), "Multi-objective optimization of material selection for sustainable products: Artificial neural networks and genetic algorithm approach", Materials and Design, Vol. 30, pp. 1209-1215. 